\title{
FILOSOFÍA, FILOSOFÍA DE LAS CIENCIAS Y LA CUESTIÓN DEL REALISMO
}

Philosophy, philosophy of sciences and the question of realism

Marcelo Díaz Soto*

Resumen

El artículo se estructura en torno a dos ejes: (1) la concepción de la filosofía como una actividad extremadamente recursiva en su aplicación al saber científico, a la ética y a la pedagogía; (2) la discusión en torno al realismo y al papel que le cabe a las representaciones en la explicación de la cognición (filosofía de las ciencias cognitivas). Se pone énfasis en mostrar la plausibilidad de los enfoques antirrepresentacionistas o constructivistas en las ciencias cognitivas, en la medida que estos enfoques pretenden superar las limitaciones y prejuicios que son propios de los enfoques computacionalistas y conexionistas que le precedieron.

Palabras clave: Recursividad filosófica, constructivismo, realismo.

Abstract

This paper consists of two main topics: (1) the conception of philosophy as an extremely recursive activity applied to scientific, ethics and educational knowledge; (2) the discussion about realism and the role of the representations in the explanation of cognition (Philosophy of Cognitive Sciences). The validity of the anti-representationism or constructivist-based perspectives, is highlighted in Cognitive Sciences where these pretend to overcome the limitations and prejudices that belong to the previous computacionalism and connectionism perspectives.

Key words: Philosophic recursivity, constructivism, realism.

\section{INTRODUCCIÓN}

Todavía a fines de la centuria pasada se enseñaba la filosofía de las ciencias como un esfuerzo individual y colectivo que había tenido sus comienzos en los años 20 en países como Alemania, Austria, Checoslovaquia y Polonia. Rudolf Carnap, Herbert Feigl, Moritz Schlick y demás miembros adscritos al neopositivismo o positivismo lógico del Círculo de Viena se consideraban como los autores que debían ser leídos y enseñados si se pretendía aprender seriamente esta disciplina filosófica. También la figura de los miembros del Círculo de Berlín como Hans Reichenbach y Richard von Mises fueron decisivos para que se constituyera la primera escuela de filosofia de las ciencias propiamente tal, esto es, con publicaciones, congresos internacionales, discípulos, etcétera. Ludwig Wittgenstein y su Tractatus Logico-Philosophicus (1921) tuvieron también un papel decisivo en la maduración de las ideas del neopositivismo al hacer posible un acercamiento entre la nueva lógica matemática y las tradiciones empiristas. 
Pero el ascenso del nacional-socialismo en Alemania y Austria trastrocó completamente la vida al interior de las universidades y del mundo de la cultura, la intelligentsia oficial pronazi nada quería saber de estos pensadores y científicos que, además de propalar ideas que cuestionaban la metafísica tradicional, especialmente la del idealismo alemán del siglo XIX, se inmiscuían en política adhiriendo a movimientos o partidos de izquierda $\mathrm{y}$, además, lo que resultaba muy provocador para las autoridades nazis, algunos de estos conspicuos pensadores y científicos eran también judíos.

La diáspora de esta comunidad por Norteamérica y Gran Bretaña tuvo como efecto que en los países de habla inglesa se difundiera el ideario de estos pensadores. De esta manera el neopositivismo, el logicismo y el fenomenalismo, entre otras posturas epistemológicas suscritas al interior del Círculo de Viena, se enriquecieron con el empirismo inglés, la filosofía analítica de Oxford, el pragmatismo, el conductismo norteamericano, etcétera.

Si bien Karl Popper había publicado su obra fundamental La lógica de la investigación científica en 1934 y en su lengua materna, el alemán, no fue sino hasta los años 50 al traducirse al inglés cuando comenzó lo que podríamos llamar una "nueva filosofia de las ciencias" para distinguirla de aquella representada por los signatarios de la llamada "concepción heredada" (received view, según la expresión introducida por Hilary Putnam). A Popper le siguieron Imre Lakatos, Thomas Kuhn, Paul Feyerabend, Wolfgang Stegmüller y varios otros autores que se explayaban con teorías acerca del método científico, las comunidades científicas con sus valores y condicionamientos históricos y sociales, la relación entre teoría y observación, etcétera. Así se llegó a fines del siglo XX con un conjunto de filosofias de las ciencias que ya habían rebasado, y muchas veces contradicho, los intereses temáticos de los filósofos y científicos de la concepción heredada. ${ }^{1}$ Junto con ello el interés por las problemáticas filosóficas que planteaban las ciencias formales y la física, que fue el núcleo de las motivaciones intelectuales explotadas por la concepción heredada, dio paso a la filosofía de la biología, a la filosofía de las ciencias sociales y a lo que llamamos con el título colectivo de ciencias cognitivas.

Es un tanto violento, me imagino, para quienes están leyendo este escrito, que no se mencione a tantos y tantos otros filósofos que habitualmente tuvimos que leer y estudiar. Quisiéramos mitigarlo solamente reconociendo que tanto los filósofos anglosajones como los europeos continentales, e incluso latinoamericanos asentados en

\footnotetext{
${ }^{1}$ Nos estamos refiriendo a la manera habitual como se han presentado los autores y temas en los cursos estándar de filosofia de las ciencias hasta los años 80 (incluidos los países de habla española). Sin embargo, hay toda una escuela de filosofia (y también de historia) de las ciencias de raigambre francesa que fue menos difundida (o que no se presentó de manera muy articulada) y que nada tiene que envidiar a la llamada visión estándar que sigue la secuencia: neopositivismo -falsacionismo popperiano o lakatiano-anarquismo metodológico- concepción estructural de las teorías científicas, etcétera. Estos autores franceses -que en algunos casos anticiparon ideas que habitualmente se ha creído que son de exclusiva creación de la llamada filosofia de la ciencia vienesa y anglosajona- son Henry Poincaré, Pierre Duhem, Emile Meyerson, Alexandre Koiré, Gastón Bachelard, Louis de Broglie.
} 
destacadas universidades del primer mundo o con un innegable reconocimiento internacional ${ }^{2}$, han contribuido para que se consolidara como un fenómeno cultural de primer orden aquello que llamamos "filosofia de las ciencias" (philosophy of sciences).

Pero la denominación "filosofia de las ciencias" empleada para identificar una disciplina filosófica no es semánticamente inocua. De hecho es necesario decir algo acerca del concepto de filosofía para no caer en una petitio de principii, si no estaríamos pasándoles de contrabando una determinada concepción de la filosofia de las ciencias (incluida la de las ciencias cognitivas) inspirada en una definición previa y no cuestionada de lo que estamos entendiendo por "filosofia". Digamos algo acerca de esto.

\section{FILOSOFÍA}

Sería pretencioso e irresponsable intentar cubrir lo que se ha entendido por "filosofia" en un par de páginas, especialmente si recordamos que una característica reconocible en la obra de los grandes filósofos que han reflexionado respecto del ser, el tiempo, el conocimiento, el espíritu, el bien, etcétera, es que ellos han sostenido una determinada concepción de lo que es la filosofia misma. No en vano hay una disciplina o subdisciplina que suele llamarse justamente "metafilosofia"3 y que da cabida a la discusión pertinente a lo que es esta actividad que tiene ya más de dos milenios en Occidente. Más fácil y rápido resulta acercarse al asunto de lo que es la filosofía simplemente constatando lo que han hecho sus cultores a lo largo del tiempo. Y lo que han hecho, al menos estructuralmente, parece incluir las siguientes fases ${ }^{4}$ : (i) hacer preguntas a propósito de un problema determinado, incluyendo, como bien nos lo han enseñado diversos filósofos, una aclaración del sentido mismo de las preguntas; (ii) proponer respuestas; (iii) justificar o dar razones que apoyen las respuestas y (iv) extraer las consecuencias de ellas. Es posible recordar que hay pensadores que escribieron voluminosas obras quedándose en la primera fase, esto es, teniendo como objetivo fundamental mostrar, por ejemplo, mediante análisis cuidadosos del lenguaje natural que la pregunta original que motivó la investigación filosófica -por ejemplo, ¿qué es la verdad?, ¿qué es lo real?, ¿qué es el significado?- carecía de sentido ${ }^{5}$. Por supuesto siempre habrá filósofos audaces, o irresponsables, que pretenderán haber cubierto todas las fases y, a su vez, habrá -salvo que se trate de sociedades totalitarias o en vías de

\footnotetext{
${ }^{2}$ Nos referinos, entre otros, a los profesores Mario Bunge (McGill University, Cánada), Miguel Espinoza (Université de Strasbourg, Francia), Ulises Moulines (Universität München, Alemania), Roberto Torreti (Universidad de Puerto Rico).

${ }^{3}$ Como la famosa publicación norteamericana The Metaphilosophy Journal.

${ }^{4}$ Ver Moulines (1991).

${ }^{5}$ La versión terapéutica o wittgensteiniana de la filosofia analítica en su momento hizo uso, y a veces abuso, de la tesis que los llamados problemas filosóficos tradicionales son enredos conceptuales.
} 
serlo- otros filósofos (o el mismo pensador en etapas diferentes de su producción intelectual) que se encargarán de hacer notar los errores del primero ${ }^{6}$.

Cuando los filósofos (de las ciencias, de la moral, de la educación, etcétera) se ponen a reflexionar, llevan a cabo de manera sistemática y "hacia el límite" una capacidad que hasta donde sabemos es exclusivamente humana, como lo ha advertido Ulises Moulines (1991) se trata de la recursividad (R.A.E.: recursivo, va, que puede repetirse indefinidamente). Piensen en el paso de baile del artista norteamericano Michael Jackson, ese movimiento en el que el bailarín parece ir retrocediendo. $\mathrm{Si}$ consideramos la realidad de una manera muy generosa como la que alberga a todo lo que hay o existe y -para tomar un caso- haciendo un recorte en lo real nos quedamos con el nivel microfísico, podemos estar de acuerdo que son los físicos de partículas quienes se relacionan con este recorte mediante sofisticados instrumentos, apoyados en complejos y especialísimos constructos teóricos y con lenguajes formales ad hoc. Pero puede ocurrir que esos mismos físicos de partículas (o algunos filósofos de la física) comiencen a reflexionar ya no acerca de ese recorte de lo real propiamente tal, sino que como el artista se van alejando y van perdiendo el contacto "más o menos directo" con lo real del que gozaba el físico de partículas ${ }^{8}$ y ahora se empiezan a cuestionar las teorías, el lenguaje y el método empleado. Si eso ocurre estamos en presencia de un físico que ahora está filosofando. Es simplemente una constatación empírica que muchos científicos de primer nivel (Galileo, Einstein, Heinsenberg, Hawking, Penrose) y muchos que les han precedido o sucedido, no pudieron (o no quisieron) soslayar sus pensamientos acerca de lo que habían creado (y no solamente en el campo teorético sino también en el metodológico). Pareciera ser que la metaciencia o filosofía de la ciencia es, como el paso de baile del artista norteamericano, algo casi inevitable cuando ya estamos poseídos por la recursividad del pensamiento filosófico.

Esto que se ha dicho respecto de la física y la filosofia de la física es también extensivo a otros campos de las ciencias empíricas y formales y también en campos en apariencia tan alejados como la moral y la ética. Veamos un caso de estos últimos: no es lo mismo desde el punto de vista de la recursividad del pensamiento el moralista o religioso que enseña o encarna valores (piénsese en Alberto Hurtado o Pierre Dubois)

\footnotetext{
${ }^{6}$ Es factible recordar que solemos hablar de un primer y un segundo (o incluso a veces de un tercer) Heidegger, Platón, Wittgenstein, entre otros, es decir, de la evolución de un pensamiento en el que no es raro encontrar en la etapa madura una o varias tesis que contradicen o superan lo que se propuso en la etapa inicial.

${ }^{7}$ Hemos querido recordar la manera cómo el filósofo chileno Jorge Millas caracterizó en su momento el quehacer filosófico (Idea de la filosofia. El conocimiento, Editorial Universitaria, Santiago de Chile, 2 volúmenes).

${ }^{8}$ Estamos conscientes de lo polémico que puede resultar afirmar que el físico especializado en el nivel microfísico tenga un contacto "más o menos directo" con lo real: hay aquí dos asuntos complejos: 1. Que el científico se conecte con lo real de una manera no completamente directa quiere decir que él observa (registra, lee) lo real mediante instrumentos muy sofisticados y no "a ojo desnudo"; 2. Que no está para nada completamente zanjada la cuestión de la naturaleza de las micropartículas (¿son entes teóricos o son efectivamente constituyentes de la realidad?).
} 
que ellos mismos u otros reflexionando acerca de la naturaleza del bien, por ejemplo. Por supuesto que en una y misma persona podemos encontrar al moralista (o sacerdote) y al axiólogo (o teólogo), como asimismo podemos encontrar al físico y al filósofo de la física o al educador y al filósofo de la educación.

Pero el físico (o el psicólogo, o el sociólogo o cualquier otro representante de las ciencias), como también el moralista (o religioso) o el educador, pueden pasárselas sin filosofar al menos conscientemente. Sin ánimo de ironizar, podemos decir que el prestigio profesional y social de cualquiera de ellos queda inalterado se planteen o no cuestiones como las siguientes: por ejemplo, para el físico, si el indeterminismo es un rasgo esencial al mundo subatómico; o para el psicólogo si la mente es un objeto complejo y problemático, cuya naturaleza hay que elucidar para ver cómo encaja en una concepción global del mundo; o para el moralista o religioso si los valores morales son objetivos o subjetivos; o para el educador si los modelos pedagógicos al uso constituyen modelos que reflejan la situación concreta del proceso educativo, o más bien si esos modelos son una idealización y simplificación de tal proceso que debe evaluarse no por su fidelidad a los hechos sino por su utilidad predictiva, etcétera. Es decir, pareciera que los diversos profesionales en el ejercicio de su actividad pueden evitar o simplemente ignorar las preguntas filosóficas, es decir, pueden marginarse de esta danza o marcha del intelecto que es, a nuestro juicio, la recursividad humana. Después de todo, nuestra admiración, por ejemplo, por la figura de Alberto Hurtado o Pierre Dubois viene por sus obras, sus compromisos y no por elucubraciones teológicas que pudieron o no ocurrir en sus cabezas.

Ahora bien, lo anterior no quiere decir que el físico, el psicólogo, el moralista o el pedagogo no incorporen en sus quehaceres respectivos supuestos filosóficos. Una cosa es marginarse (conscientemente o no) de la reflexión filosófica y otra muy distinta es que la filosofia no esté efectivamente presente en el quehacer científico, espiritual o pedagógico. Ya sabemos que el talento en una determinada actividad no necesariamente va acompañado de sensibilidad filosófica. Por ejemplo, el realismo ${ }^{9}$, es una filosofía (epistemología) que está en la base de gran parte de la ciencia natural contemporánea, pero no abundan los científicos conscientes de que esa filosofía inspira o subyace en su quehacer disciplinar. Sin embargo, no es posible que un científico (o tecnólogo) analice una muestra química si no está, tácitamente, admitiendo que hay un mundo externo del que forman parte los componentes químicos que estudia y que ese mundo es cognoscible en parte, $\mathrm{y}$ gradualmente, por medio de las hipótesis, teorías y experimentos que él y sus colegas suscriben y realizan.

Quizás ustedes, estimados lectores, pensarán que estamos tomando posición a favor del realismo en alguna de sus expresiones, es decir, que se está haciendo filosofía

\footnotetext{
${ }^{9}$ El realismo ha tenido diversas expresiones a partir de la segunda mitad del siglo XX. Tenemos el realismo hipotético (Konrad Lorenz, Gerd Vollmer y demás partidarios de la epistemología evolucionista); realismo interno o pragmático (Hilary Putnam); realismo científico (Mario Bunge, Ian Hacking, Wilfrid Sellars); realismo metafísico (Miguel Espinoza), etcétera.
} 
(epistemología) a partir de una situación generada en una ciencia particular (química). Pero, si ese fuera el caso, ¿no es ello acaso un síntoma de que la filosofía está en la base y prolongación racional de las ciencias? Esta es una tesis, metafilosófica por cierto, que uno debería estar dispuesto a defender especialmente en estos tiempos en que la filosofia -al menos en algunas de sus expresiones- parece estar alejada de la racionalidad que nos legó el pensamiento moderno.

Por otro lado, es verdad que el positivismo, sea en su versión comteana (siglo $\mathrm{XIX}$ ) o vienesa (siglo XX), quedó relegado a poco más que una materia de pregrado que hay que digerir para comprender el desarrollo de la filosofía de las ciencias. El cuestionamiento de esa corriente de pensamiento llegó de varios frentes, por un lado la propia evolución de las ideas de algunos connotados partidarios del neopositivismo, entre ellos Friedrich Waismann con su denuncia del carácter metafísico del propio principio de verificabilidad y Rudolf Carnap con el desarrollo de la lógica probabilitaria; por otro lado, las críticas de pensadores norteamericanos a varias afirmaciones de la "concepción heredada", entre ellas la crítica a la distinción analítico/sintético por parte de Willard van Orman Quine y el propio Putnam, la discusión en torno a los condicionales contrafácticos y a la tesis de la extensionalidad de las leyes científicas debida a Nelson Goodman y Roderick Chisholm, etcétera.

Súmese a lo anterior las críticas globales al positivismo que llegaron de la llamada "filosofía continental", así como lo hizo la Escuela de Frankfurt, y nos daremos cuenta que la concepción heredada ya en los años 60 estaba en crisis. Incluso es posible consignar en este brevísimo recuento que otro golpe, generado esta vez fuera de la filosofía, fue el impacto que tuvo la psicología de la gestalt en las reflexiones acerca del método científico. En la obra madura de Karl Popper, en los escritos de Thomas Kuhn, de Paul Feyerabend y de Norwood Russell Hanson, se puso en la picota -gracias a la inspiración que estos pensadores recibieron de los estudios sobre la percepción- la creencia en que en el método científico está operando una observación pura. De esta manera el empirismo y fenomenismo de la concepción heredada quedaba en entredicho.

\section{EL COGNITIVISMO Y SUS FILOSOFÍAS}

A mediados de los años 70 se consolidó el paradigma cognitivista y qué duda puede caber con que es un paradigma afianzado, con una aplastante presencia en lo cotidiano, al punto que nos hemos acostumbrado a considerarnos inmersos en una "sociedad del conocimiento o de la información". Lo curioso es que se supone que entendemos muy bien la expresión, especialmente porque de facto muchísimas personas a diario se comunican mediante los diversos recursos informáticos que hay a mano (Facebook, WhatsApp, Twitter y otras futuras redes infosociales).

Pero en el uso de estos recursos tecnológicos están comprometidas las responsabilidades individuales y colectivas como tan dramáticamente expuso Edward Snowden, generando una crisis política, diplomática y de seguridad de grueso calibre. 
Para qué hablar más de los efectos nocivos de la infoadicción (como las redes de pederastas y otros crímenes informáticos), quedémonos solamente con la parte más amable, por ejemplo con el acceso barato e inmediato a mucha información contenida en bibliotecas virtuales, con la transacción económica rápida y eficaz entre contratantes lejanos, etcétera. También están los efectos ambivalentes originados en el hecho de que la tecnología de la información no trata del significado o contenido de los mensajes, sino que trata del procesamiento y transmisión de ellos. Recordemos la interesante discusión que hace algunas décadas suscitaron filósofos de distinto signo, como el analítico John Searle y el heideggeriano Hubert Dreyfus ${ }^{10}$, a propósito del cuestionamiento de la inteligencia de las máquinas.

Pero ¿cómo es que se llegó a replantear la cuestión de que las máquinas pudiesen pensar, ser inteligentes, en el contexto de este paradigma que se origina en los años 40 ? Ya sabemos que esto tiene que ver con la emergencia de la informática y la figura del matemático inglés Alan Turing y otros destacados precursores del llamado paradigma cognitivista. Digamos algo acerca de este punto abordándolo desde una perspectiva familiar.

Una creencia de sentido común es que usted, yo y los demás individuos poseemos funciones cognitivas, es decir, que pensamos, calculamos, creemos, recordamos, etcétera; junto con ello, y a partir del siglo XIX cuando ya estaba completamente superada la hipótesis cartesiana del animal-autómata, incluimos a los animales superiores dentro de esta categoría de entes con funciones cognitivas. Pero a las máquinas propiamente tales, casi por un acto reflejo las considerábamos fuera de este grupo de las entidades con funciones cognitivas. En este sentido Turing a comienzos de los años 50 fue inmensamente provocador cuando planteó la posibilidad de alterar esta distinción de sentido común mediante dos grandes contribuciones: una inspirada en la psicología conductista, el llamado test de Turing, y el otro en la concepción de un dispositivo mecánico programable y universal (la máquina universal de Turing). El primero planteaba que si una máquina puede imitar el comportamiento inteligente de una persona, al punto que un observador externo no fuera capaz de precisar si la respuesta que ha recibido a la pregunta que él ha realizado la ha dado una persona o una máquina, entonces, eso debe tomarse como una evidencia de que la máquina es capaz de pensar (jla tesis de l' homme machine de Julien La Mettrie había resucitado!). El segundo imaginaba un dispositivo mecánico e ideal de cálculo capaz de resolver una función matemática que sea computable y se considera que una función es computable cuando su solución es susceptible de ser obtenida por un procedimiento mecánico.

Con Turing y la contribución colectiva de especialistas en ingeniería (Shanon), psicología (Lashley, Miller, Brunner), matemáticas (von Neumann, Wiener, McCarthy, Minsky), neurología (Mc Culloch), lógica (Pits), lingüística (Chomsky), especialistas en computación (Simon, Newell, Johnson Laird) la mesa quedó servida. Los comensales tuvieron que sazonar adecuadamente lo que tenían enfrente para constituir un influyente

\footnotetext{
${ }^{10}$ John Searle (1980) y su famosa parábola de la sala china en "Minds, Brains and Programs" (p. 417-424), y Hubert Dreyfus (1972), en What Computers Can't Do. A Critique of Artificial Reason.
} 
paradigma que, como lo mencionamos anteriormente, a mediados de los 70 quedó consolidado, se transformó en lo que Kuhn llamaría un paradigma en su etapa de "ciencia normal".

El desafío que dejó Turing, esto es la posibilidad de tener máquinas inteligentes, recibió varias respuestas. En cierto sentido puede decirse que el conjunto de estas respuestas es, en parte, la historia de las ciencias cognitivas, la historia de este esfuerzo interdisciplinario por centrarse en el estudio de la cognición, los procesos cognitivos, la mente humana, la mente del animal, el procesamiento de la información, la inteligencia artificial (Emilio García, 2001) ${ }^{11}$.

En una apretada (y seguramente un tanto arbitraria) taxonomía es posible reconocer las siguientes principales etapas o respuestas al desafío de Turing ${ }^{12}$ :

$1^{\text {a }}$ respuesta: Cognitivismo clásico o computacionalista (=informativista, inteligencia artificial fuerte, modelo de procesamiento de símbolos). Representantes: Noam Chomsky, Alan Newel, Herbert Simon, Zenon Pylyshyn, el primer Hilary Putman, Jerry Fodor, etcétera.

En esta respuesta se elimina la distinción entre objetos de los que predicamos funciones cognitivas (OFC) y objetos de los que las negamos $(\mathrm{O}-\mathrm{FC})^{13}$ y se acepta el desafío con todas sus consecuencias. Entonces los OFC incluirían las mentes y las máquinas. Este es el punto de vista ortodoxo, muy en la línea de Turing. La idea central aquí sería que las máquinas son procesadores de símbolos. El enfoque filosófico que subyace aquí es el funcionalismo.

$2^{\mathrm{a}}$ respuesta: Proviene de la psicología cognitiva y está representado por autores como A. Paivio, R. Shepard, S. Kosslyn. Consideran el desafío de Turing como una metáfora. Se acepta el lenguaje informático, pero se evitan las consecuencias polémicas

\footnotetext{
${ }^{11}$ Por supuesto que esta manera de caracterizar a las ciencias cognitivas no es la que suscribirían todos los llamados científicos cognitivos. Se podría también caracterizar de una manera muy sucinta diciendo que las ciencias cognitivas tratan de entidades que poseen cognición, asumiendo que estas entidades son, o pueden ser, tanto sistemas naturales (organismos, personas) como sistemas artificiales (máquinas). Sin embargo, no es tan simple la cuestión de la definición. Por ejemplo, Francisco Varela (2005) en su manual Cognitive Science. A Cartography of Current Ideas, prefería hablar de ciencias y tecnologías de la cognición para no perder de vista que son un híbrido de disciplinas y tecnologías en las que no es posible distinguir entre ciencia pura y ciencia aplicada. Por su parte el Diccionario de Ciencias Cognitivas de Olivier Houdé (2003), Daniel Kaiser et al. nos dice que las ciencias cognitivas son un campo del saber que intenta dilucidar mediante la experimentación, la modelización y el uso de tecnologías de punta, el Misterio de la Mente en sus relaciones con la Materia: el cerebro, el cuerpo y la computadora. Es factible destacar en que en los dos casos se reconoce el papel fundamental que tiene la filosofía (filosofia de la mente y epistemología) en el planteamiento y tratamiento de los temas y problemas que caracterizan a las ciencias cognitivas.

${ }^{12}$ Una versión más simple de esta taxonomía se encuentra en M. Díaz y A. Espinoza (2009, p. 119-133).

${ }^{13}$ El psicólogo español Ángel Riviére (1991) prefiere hablar de FM (entidades con funciones mentales) y FM (entidades sin funciones mentales). Nos parece que es más ilustrativo hablar de OFC y O-FC para no olvidar que entre la amplia gama de funciones, propiedades o rasgos que asociamos a lo mental (sentimientos, percepciones, recuerdos, voliciones, etcétera), estamos fundamentalmente hablando de aquellas funciones que son primariamente de carácter cognitivo.
} 
del cognitivismo clásico. Es un enfoque pragmático. No se comprometen con el enfoque de Turing.

$3^{\text {a }}$ respuesta: cognitivismo no clásico o conexionismo ( $=$ modelo de redes neurales, procesamiento distribuido en paralelo). Algunos representantes son D. Ballard, G. Hinton, D. Zipser.

En este caso la respuesta al desafio de Turing se considera que los procesos cognitivos son cambios cuantitativos que se da en los estados de activación de las redes neurales. Se rechaza homologar los procesos cognitivos a modelos de cómputos; son más bien modelos idealizados en el computador de cómo actúa el cerebro, esto es, a la manera de una red.

Los filósofos más proclives a los modelos conexionistas han sido Paul Churchland y Patricia Smith, ellos se han inclinado por un rechazo radical de todo mentalismo -y con ello de la psicología de sentido común o popular-, como se encuentra en el modelo cognitivo clásico representado, entre otros, por Fodor. La postura filosófica de estos autores es materialista y se llama Eliminativismo.

$4^{\mathrm{a}}$ Respuesta. Es la de John Searle y otros autores como Mario Bunge que se pueden considerar representantes de un enfoque materialista y emergentista ("naturalismo biológico" prefiere llamarlo Searle) o incluso de cuño heideggeriano (como Hubert Dreyfus). Se mantiene la distinción entre OFC y O-FC, pues se considera que ni las máquinas piensan ni las mentes son máquinas. El pensar se considera exclusivo de los seres vivos.

$5^{\text {a }}$ Respuesta. Es la más influyente en estos momentos porque no está circunscrita al ámbito de especialistas en ciencias cognitivas, sino que también está presente en las teorías pedagógicas, es decir, ha circulado desde la psicología y la biología hacia lo pedagógico con el nombre bastante amplio de "constructivismo". ${ }^{14} \mathrm{En}$ nuestro medio, la llamada "Escuela chilena" de Humberto Maturana y Francisco Varela, en los años 70, proponen el concepto de autonomía biológica y autopoiesis en el contexto de la biología teórica. Más tarde la Teoría de la Enacción de Varela plantea la continuidad entre la vida y la mente, de esta manera las ideas tradicionales de las ciencias cognitivas que se incluyen en las respuestas anteriores (cognitivismo computacionalista y conexionista), entre ellas las ideas del procesamiento de la información y de las representaciones mentales, dejan paso a una visión en la que los organismos adquieren un rol activo en la configuración de su experiencia. Varela (2009) va a reconocer generosamente en su obra El fenómeno de la vida la deuda intelectual con la fenomenología, especialmente con Maurice Merleau-Ponty, Edmund Husserl y Martin Heidegger. Mención aparte, pero siempre dentro de esta generosa diversidad que es el constructivismo, se encuentra la propuesta de la cognición (o mente) corporeizada de George Lakoff y Mark Johnson (2003, 1999), quienes también reconocen la

\footnotetext{
${ }^{14}$ Me temo que la evaluación epistemológica del constructivismo no se ha realizado de manera sistemática, salvo en autores muy reputados que efectivamente se pueden considerar como los precursores del constructivismo, este es el caso de Jean Piaget y Francisco Varela.
} 
impronta de la fenomenología de Merleau-Ponty y postulan que la cognición es el producto de proyecciones metafóricas estrechamente enraizadas en la experiencia ${ }^{15}$.

En términos generales, podemos decir que el paradigma cognitivo se ha consolidado, se ha institucionalizado, se ha propagado, y hoy tenemos legiones de especialistas en biología y neurociencia, lingüística, psicología, informática, etc., que diseñan, investigan, enseñan, publican y piensan imbuidos de modelos cognitivos que se han ido desarrollando al alero de los cambios que han afectado a este paradigma. Si siguiéramos en parte el esquema que Kuhn (1962) propuso en su famoso libro The Structure of Scientific Revolutions para explicar el desarrollo de las ciencias, tendríamos que reconocer que este paradigma se encuentra en su etapa de ciencia normal, encontramos ámbitos en los que el paradigma funciona (ejemplos paradigmáticos), es decir, permite explicar, controlar y simular procesos tan complejos como los cognitivosrepresentacionales-intencionales que, en el mundo animal y humano, están ligados con la percepción y la acción.

En contra de la opinión de autores como Husserl, que identifican la intencionalidad con la propiedad de un acto de representación consciente, en las ciencias cognitivas el concepto de intencionalidad es considerado independiente de toda toma de conciencia de un contenido de pensamiento. Pero justamente la conciencia o el carácter subjetivo de la experiencia consciente (conciencia fenomenológica), presentan un formidable desafio a las ciencias cognitivas actuales. En términos kuhnianos nos encontramos con una anomalía, un ámbito donde el paradigma no funciona. Se trata de una brecha explicativa, en el sentido que no disponemos de una teoría convincente y suficientemente testeada acerca de nuestra naturaleza fisica o funcional que explique nuestra experiencia subjetiva. Sin embargo, no podríamos decir que tal traspié sea, por el momento, indicador de una crisis del paradigma, al menos en el sentido que los cognitivistas estén atrapados en un escepticismo sistemático y terminal.

Ahora bien, que el mundo esté ahí con sus estados de cosas estructurados e independientes del sujeto (realismo) no es una creencia que encontremos así, tan limpiamente, en la biología y en la psicología de la cognición. Por el contrario, como vimos anteriormente ( $5^{\mathrm{a}}$ Respuesta al desafio de Turing) el constructivismo ha reactualizado un enfoque epistemológico de tipo antirrealista. Detengámonos un tanto en este punto.

Los trabajos de Jean Piaget son una de las fuentes esenciales del actual constructivismo interdisciplinario que encontramos en las ciencias cognitivas (véase $\mathrm{O}$. Houdé, D. Kayser et al, 2003, 101-103). Piaget se oponía tanto al innatismo como al

\footnotetext{
${ }^{15}$ Lakoff y Johnson $(2003$, 1999) piensan que el conocimiento se origina en unidades básicas de la percepción, que resultan de la experiencia espacial y temporal de nuestros propios cuerpos dotados de cierta dinámica. Santiago Castillo ha escrito al respecto que "en todo esto hay un argumento de fondo, el cual es que la experiencia entrega las pautas (o esquemas) que hacen posible el desarrollo del conocimiento. Esto concuerda plenamente con los planteamientos de Varela sobre mente corporizada". Véase S. Castillo, "Emergencia y mente corporizada en el problema mente-cuerpo. Causalidad a diferentes niveles". Tesis de Magíster en Filosofia de las Ciencias, Universidad de Santiago de Chile.
} 
empirismo y describe la inteligencia como la forma más general de las coordinaciones de las operaciones y acciones de un sujeto que se construye (re)construyendo, (re)estructurando lógicamente su entorno. Para el constructivismo el conocimiento no es el reflejo del mundo exterior, ni es la proyección acerca de la realidad de estructuras trascendentales innatas de la mente. Es admisible reconocer en esto que se reactualizan viejas disputas filosóficas, epistemológicas, que tiñeron la modernidad. El mundo físico se debe modelizar, "reiventar" (Paul Watzlawick), "hacer emerger" (Francisco Varela), es decir, que solo en virtud de las acciones y operaciones que el sujeto ejerce sobre el mundo físico es que lo reconoce recortado en objetos singulares interrelacionados ${ }^{16}$.

Ahora bien, ser sensible a los supuestos filosóficos (epistemológicos y ontológicos principalmente) de la ciencia contemporánea, es ser consciente de los peligros que una mala filosofía (una ontología o una epistemología defectuosa) pueda generar al colarse, por ejemplo, en un programa o línea de investigación científica. Una cosmovisión que no cumpla requisitos mínimos de racionalidad (conceptual, lógica, metodológica, epistemológica, ontológica, entre otros $)^{17}$ nos llevará indefectiblemente a perdernos en discusiones estériles o, peor aún si se piensa en el bien colectivo, a dilapidar recursos económicos y humanos en proyectos que, de haber sido examinados (discutidos) en sus presupuestos filosóficos, se habría, como mínimo, sospechado que estaban condenados al fracaso.

Es cierto que ha habido momentos - como nos lo ha enseñado la historia de las ciencias- en los que un campo de investigación novedoso y fructífero incluyó originalmente motivaciones extracientíficas, como las de naturaleza mística o artística (valga como ejemplo clásico las motivaciones esotéricas-religiosas de Johannes Képler $(1571-1630)^{18}$, que se combinaban con un riguroso apego a los hechos observados y comprobados. Pero estos momentos, que podríamos llamar "pre-paradigmáticos", siguiendo otra vez la terminología de Thomas Kuhn en su explicación del desarrollo de las ciencias, dieron pie más tarde al reconocimiento que más allá de estos desvaríos

\footnotetext{
${ }^{16}$ El constructivismo radicalmente pragmático de Varela (conocido como "enfoque enactivo", inspirado en los modelos de autómatas celulares aplicados a la neurofisiología de la percepción, cuestiona abiertamente el criterio de evaluación de la cognición sustentado en el enfoque cognitivo clásico, criterio que es el de la representación adecuada de un mundo exterior predeterminado. Varela va más allá de las ciencias cognitivas y apunta a cuestionar la filosofia de la representación. Si bien Varela reconoce que en la tradición francesa y alemana (Maurice Merleau-Ponty. Michel Serres. Michel Foucault, Martin Heidegger, etcétera) se encuentran importantes cuestionamientos a tal filosofía, no es descaminado precisar que tal cuestionamiento se encuentra también en Ludwig Wittgenstein y quizás en John Dewey (véase acerca de esto último la tesis general defendida por Richard Rorty en La filosofia y el espejo de la naturaleza, 1983).

${ }^{17}$ La racionalidad conceptual apunta a minimizar la imprecisión; la lógica a evitar la contradicción; la metodológica a cuestionar, dudar o criticar, y justificar (exigir demostración o datos, favorables o desfavorables); la epistemológica o gnoseológica a valorar el apoyo empírico y evitar conjeturas incompatibles con el grueso del conocimiento científico y tecnológico; la ontológica a adoptar una concepción del mundo coherente y compatible con el grueso de la ciencia y la tecnología vigente. Véase M. Bunge (1988).

${ }^{18}$ Véase Desiderio Papp (1975).
} 
extracientíficos, se encontraban sólidos constructos teóricos y líneas de investigación ${ }^{19}$. Se podría decir que las creencias que colisionan con la racionalidad ontológica o la racionalidad epistemológica no son per se un presente griego para la ciencias, el peligro que tales creencias representan radica más bien en su permanencia y ramificación, cual patógeno en un cuerpo, en el seno de un constructo teórico que ha sido adoptado por una comunidad de especialistas. La tarea de quien reflexione respecto de la ciencia es, en parte, la de detectar y eliminar o neutralizar esas creencias poniendo a la vista su contradicción con el resto del corpus científico. Por ejemplo, si en algún enfoque pedagógico se detecta un peligro de esta índole habría que mostrar cómo tal creencia entra en conflicto con las teorías más sólidas en las disciplinas que alimentan a la pedagogía (la psicología, sociología y otras).

\section{LAS REPRESENTACIONES Y LA CUESTIÓN DEL REALISMO}

Finalmente, en esta última sección trataremos dos de los tópicos que resultan caro a los enfoques cognitivistas, especialmente al constructivista, se trata de la cuestión de la naturaleza y función de las representaciones y el cuestionamiento que se hace a toda postura realista. Como sabemos los constructivistas han hecho de este asunto su punta de lanza para distanciarse de la primera y tercera respuesta al desafío de Turing. El mote de antirrepresentacionistas no es injusto con los puntos de vista que ellos nos han mostrado. Tómese nota del siguiente pasaje de un pasaje de El Fenómeno de la Vida de Varela (2000):

Queremos cuestionar este hábito inveterado, como se manifiesta explícitamente en los estudiantes de la neurociencia. Lo llamamos el programa representacionista. Tiene sus raíces en un complejo de ideas que se tornaron visibles en su actual forma con el surgimiento de la ingeniería de las comunicaciones y la ciencia computacional, pero que es una herencia mucho más antigua en las preferencias anglosajonas. El sistema nervioso captura características del entorno y construye representaciones del mundo en el que vive el animal con el fin de utilizarlas como acciones de adaptación del organismo (p. 154).

Más adelante en la misma obra Varela escribe en términos contundentes lo siguiente:

La total ausencia, hasta ahora, de sentido común en la definición de cognición, constituye la principal fuente de insatisfacción de la llamada alternativa de la Enacción. Tanto en el cognitivismo (por su misma base) como en el conexionismo de nuestros días (por la manera en que se lo practica), todavía se observa que los criterios para la cognición están directamente relacionados con la representación exitosa de un mundo externo, dado previamente, normalmente como una situación que resuelve un problema. Sin embargo, nuestra actividad cognitiva en la vida diaria

\footnotetext{
${ }^{19}$ Este es el caso de las sólidas contribuciones científicas de Képler, las que fueron finalmente reconocidas en el siglo XIX al reeditarse sus obras que estaban casi desaparecidas.
} 
revela que esta visión de la cognición es demasiado incompleta. Precisamente, la principal habilidad de toda cognición viviente es, dentro de límites amplios, proponer los temas relevantes que han de abordarse en cada momento de nuestras vidas. No son pre-dados, sino activados o traídos a un primer plano desde un contexto y lo que cuenta como relevante es lo que nuestro sentido común determina como tal, siempre de una manera contextual (2000, p. 203).

Es difícil no reconocer en estos pasajes el parecido con reflexiones que se encuentran en varios filósofos contemporáneos que con pasión han participado en la discusión de que la cognición sea un proceso de representación de un mundo o estado de cosas pre-dado, estructurado y preexistente. Para ellos la cuestión acuciante y que vale traer a colación una vez más es la pregunta de por qué el realismo referido al mundo externo se ha transformado en un problema. Para los filósofos de la modernidad, como bien sabemos, los objetos inmediatos que percibimos no son objetos físicos, son objetos mentales. El filósofo Hilary Putnam (1999), al igual que Varela como vimos en la primera cita de El fenómeno de la Vida, se ha fijado también en que en las ciencias cognitivas se ha colado la hipótesis acerca de la existencia de representaciones en nuestra computadora cerebral. Si asumimos, escribe Putnam, que la mente es un órgano e identificamos la mente con el cerebro, entonces se hace inevitable:

1.Pensar en algunas de las "representaciones" como análogas a las "impresiones" de los filósofos clásicos (la computadora cerebral o la mente haría inferencias a partir de algunas representaciones, estas inferencias serían el resultado del proceso perceptivo de la misma manera que en la versión clásica la mente realiza inferencias a partir de las impresiones) y

2.Pensar que estas "representaciones" están unidas a los objetos que están en el entorno del organismo solamente de manera causal y no cognitivamente (de la misma manera que las impresiones estaban unidas a los "objetos externos" solamente de manera causal y no cognitiva (Putnam, 1999, p. 9-10).

El tratamiento de la cuestión del realismo ha dado lugar a diversos "ismos" (realismo directo/indirecto científico o de sentido común, realismo natural, etcétera) dentro de lo que, al menos en la epistemología contemporánea, se denomina como "teorías filosóficas de la percepción". Lo que hemos hecho al traer a colación a Putnam, o al mismo Varela, es destapar una caja filosófica de Pandora y no es aquí el lugar para acometer la magna tarea de individualizar cada una de las sorpresas que nos depara la caja abierta.

Seremos mucho más modestos acercándonos al problema del realismo y de las representaciones mediante una situación hipotética como la siguiente. Imagínese que en este preciso momento ingresa al lugar en que están leyendo este artículo un ser que, a todas luces, no es un terrícola. Una pregunta que le hacemos a usted es si podemos abrigar la esperanza de comunicarnos con ese extraterrestre. Parece ser que como ignoramos en qué consiste su sistema de representación, no tenemos una respuesta a mano. Si, en cambio, tuviéramos información acerca de su constitución interna, de qué 
está hecho, si tiene algo parecido a un cerebro y a un sistema nervioso central, etcétera, nuestra esperanza de tener contacto con él tendría una base razonable ${ }^{20}$. Pero fijense que en esta situación hipotética que les hemos planteado está operando un arraigado prejuicio epistemológico: que para que haya cognición propiamente tal tiene que haber representaciones (sean imágenes, ideas, símbolos).

Cuando nos fijamos en la tradición moderna, especialmente en la empirista, nos damos cuenta que la mayoría de sus más influyentes pensadores desarrollaron elaboradas teorías acerca de la naturaleza, función y origen de las representaciones. En este sentido, cuando autores antirrepresentacionistas como Varela se rebelan en contra de este prejuicio, uno no puede dejar de pensar que una vieja discusión ha resurgido como el ave fénix de la mano de las actualísimas ciencias cognitivas. Pero de todas maneras hay que tener presente que las discusiones entre representacionistas y antirrepresentacionistas va a depender en parte de lo que se entienda por "representación".

Pero ¿qué implica la noción de representación? Un primer rasgo es que implica la idea de representación de algo real. Cómo ocurra esto es ya parte del arduo y complejo debate. Un segundo rasgo es que la representación mantiene un rasgo equivalente con lo real, es funcionalmente adecuada. Pero es funcionalmente adecuada solo si se da en el marco de un sistema cognitivo, así como lo mostramos en la Figura 1 que viene a continuación: ${ }^{21}$

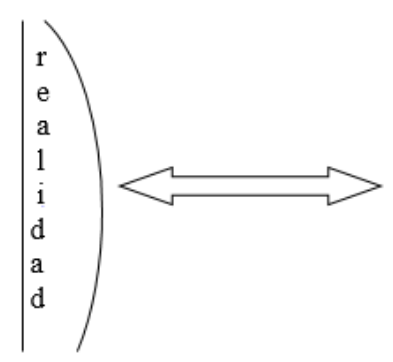

Sistema Cognitivo

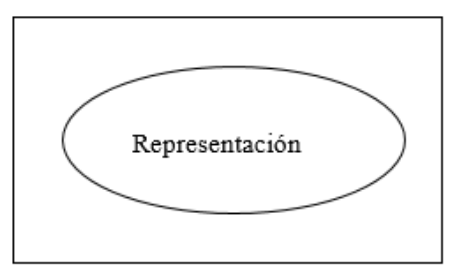

Pero ¿cuándo podemos decir que nuestro sistema de representación es adecuado? Respuesta: cuando, por ejemplo, podemos movernos sin chocar con las cosas podemos decir que estamos en un sistema adecuado, nuestras representaciones son funcionalmente adecuadas.

Ahora bien, una representación es funcionalmente adecuada cuando es viable o sirve. En este sentido puede decirse que no sabemos lo que tienen en común la representación de una mesa, por ejemplo, en sistemas cognitivos distintos como un murciélago, una persona o quizás en un extraterrestre. Por tanto, la adecuación

\footnotetext{
${ }^{20}$ Recuerden la película Encuentros cercanos del tercer tipo (1977) de Steven Spielberg. En ella el contacto entre humanos y una civilización alienígena se daba por medio de notas musicales.

${ }^{21}$ Rememoramos, en la figura y en el comentario que le sigue, las interesantes enseñanzas del profesor Álvaro Moreno Bergaretxe a mediados de los 90 en la Universidad del País Vasco.
} 
funcional o equivalencia se plantea siempre en el marco de un sistema cognitivo determinado. Si se tratara de un murciélago y este chocara con la mesa, diríamos que no tiene una representación adecuada de la mesa.

Pero la equivalencia funcional no es un mapa o un mero reflejo idéntico de algo, es decir, no hay isomorfismo, sino que hay que remitirse al código interno de representación, esto es, al marco del sistema cognitivo. Finalmente, si insistimos en la magna cuestión de qué es el mundo real, estaremos frente a un problema fundamental. Pero la pregunta acerca de lo que es la representación es abordable si se entiende por tal un procedimiento operacional, así parece que evitamos el espinudo asunto de lo que son las representaciones. Sin embargo, seguimos suponiendo entonces que hay un mundo real. Parece que nos hemos pisado la cola. ¿Y nuestro extraterrestre creerá que todo lo que le rodea es parte del mundo real?, ¿cómo podríamos saber si tiene un sistema de representaciones que sea funcionalmente adecuado?, ¿podemos evitar la antropomorfización a la hora de evaluar al extraterrestre con sus eventuales capacidades cognitivas?

Universidad de Santiago de Chile*

Facultad de Humanidades

Departamento de Filosofia Alameda Bernardo O'Higgins 3363. Santiago (Chile) marcelo.diaz.s@usach.cl

Este artículo forma parte del proyecto de Investigación DICYT, USACH, cód. 031553DS (20152016) y del Proyecto Mayor de Investigación UTA, cód. 3735-16 (2016-2017).

\section{OBRAS CITADAS}

Bunge, Mario (1988). Racionalidad y realismo. Madrid: Alianza.

Díaz, Marcelo \& Espinoza, Alex (2009). "Las ontologías del paradigma cognitivo: computacionalismo, conexionismo y materialismo emergentista". Alpha, 28 (julio):119-133.

Dreyfus Hubert (1972). What Computers Can't Do. A Critique of Artificial Reason. New York: Harper and Row.

García, Emilio (2001). Mente y Cerebro. Madrid: Editorial Síntesis.

Houdé, Olivier; Kayser, Daniel et al. (2003). Diccionario de Ciencias Cognitivas. Buenos Aires: Amorrortu.

Kuhn, Thomas (1962). The Structure of Scientific Revolutions. Chicago: University Chicago Press.

Lakoff, George; Johnson, Mark (2003). Metaphors we live by. Chicago:University of Chicago Press.

_ (1999). Philosophy in the flesh. The embodied mind and its challenge to western thought. New York: Basic Book. 
Moulines, Ulises (1991). Pluralidad y recursión. Estudios epistemológicos. Madrid: Alianza. Papp, Desiderio (1975). Ideas revolucionarias en la ciencia. Santiago de Chile: Editorial Universitaria, tomo I.

Piaget, Jean (1954). The Construction of Reality in the Child, New York; Basic Books.

Putnam, Hilary (1999). The Threefold cord. Mind, Body, and World. New York: Columbia University Press.

Riviére, Ángel (1991). Objetos con mente. Madrid: Alianza.

Rorty, Richard (1983). La filosofia y el espejo de la naturaleza. Madrid: Cátedra.

Searle John (1980). "Minds, Brains and Programs", Behavioral and Brain Science 3: 417- 424.

Varela, Francisco (2005). Conocer. Las ciencias cognitivas: tendencias y perspectivas. Cartografia de las ideas actuales. Barcelona: Gedisa.

- (2000). El fenómeno de la vida. Santiago de Chile: Dolmen Ediciones.

Varela, Francisco; Thompson, E. and Rosch, E. (1991) The Embodied Mind: Cognitive science and human experience, Cambridge: MIT Press. 